\title{
Return to the Original Work Activity Following the Full- endoscopic Lumbar Surgery under the Local Anesthesia
}

\author{
Makoto TAKEUCHI, ${ }^{1}$ Kazuta YAMASHITA, ${ }^{1}$ Ayaka HASHIMOTO, ${ }^{1}$ \\ Nobutoshi TAKAMATSU, ${ }^{1}$ Kosuke SugIURA, ${ }^{1}$ Hiroaki MANABE, ${ }^{1}$ \\ Fumitake TEzUKA, ${ }^{1}$ Yoichiro TAKATA, ${ }^{1}$ Toshinori SAKAI, ${ }^{1}$ Toru MAEDA, ${ }^{1}$ \\ and Koichi SAIRYO ${ }^{1}$ \\ ${ }^{1}$ Department of Orthopedics, Tokushima University Graduate School, Tokushima, \\ Tokushima, Japan
}

\begin{abstract}
Transforaminal full-endoscopic spine (TF-FES) surgery is minimally invasive and can be performed under local anesthesia. Thus, it is expected that the patient can return to work (RTW) quickly. However, information in the literature regarding this is sparse. The purpose of this study is to review the timing of RTW after TF-FES surgery. This study involved 50 patients (14 women, 36 men; mean age 44.5 years, age range: $20-65$ years) who underwent TF-FES surgery between January 2016 and April 2018. All the patients were active workers. Occupations varied widely (e.g., physician, nurse, helper, clerk, construction worker, chef, and schoolteacher). There were no surgery-related complications. Median time to RTW was 21 days. More than half of the patients could RTW within 21 days. In all, 12 cases (24\%) could have RTW within 7 days. Occupations of 12 patients who achieved RTW within 7 days included physician, company owner, and restaurant owner, with 11 in the Light work, 1 was in the Medium work, and none in the Heavy work. All 12 had a quick RTW because their work was Light and they could not take prolonged sick leave. Prompt RTW is possible with TF-FES surgery. The biggest merit of TF-FES surgery is minimal invasiveness to the muscles of the back. Also, it can be performed under local anesthesia. Our findings reveal quicker RTW after surgery, depending on occupational type.
\end{abstract}

Keywords: full-endoscopic endoscopic surgery, local anesthesia, return to work

\section{Introduction}

The gold standard treatment for lumbar herniated nucleus pulposus (HNP) and for lumbar spinal stenosis (LSS) without slippage is posterior decompression, including discectomy and laminectomy, under general anesthesia. The Royal College of Surgeons recommendation is to achieve return to work (RTW) at about $4-6$ weeks after discectomy. ${ }^{1)}$

Transforaminal full-endoscopic spine (TF-FES) surgery is minimally invasive and can be done under local anesthesia. ${ }^{2-5)}$ The procedure requires only an 8-mm skin incision, and damage to the muscles of the back is minimal. It is currently the minimally

Received June 25, 2020; Accepted October 21, 2020

Copyright $\odot 2021$ by The Japan Neurosurgical Society This work is licensed under a Creative Commons AttributionNonCommercial-NoDerivatives International License. invasive spine surgery procedure, and thus it is expected that patients can return to their original work activity quickly. However, information in the literature in this regard is sparse.

In this study, we reviewed speed of RTW following TF-FES surgery in relation to various occupational types and analyzed the factor affecting the time to RTW.

\section{Materials and Methods}

This study involved 50 patients (14 women, 36 men; mean age 44.5 years, age range: 20-65 years who underwent TF-FES surgery between January 2016 and April 2018. All were active workers, and any patients who had retired from active service were excluded. This research has been approved by the institutional ethics review board of the authors' affiliated institutions. 
The TF-FES procedure included discectomy for HNP and decompression for LSS. Those surgeries were conducted under local anesthesia by four surgeons who specialized in spinal surgery. During the study period, TF-FES surgery was usually performed with the "outside-in procedures" that were done by placing the cannula on the disc surface and just outside of the intervertebral foramen. In that procedure, by widening the foramen by high speed drill, safe insertion of cannula was possible without pushing on the exiting nerve root. The outside-in procedure is described in detail in a paper by Yoshinari et al. ${ }^{6)}$

The patients routinely started standing and walking 1-2 hours after surgery. Closed suction drains were used after all of TF-FES surgery and removed the day after surgery. They were allowed to be discharged the 1-3 days after the surgery. Return to light work such as office work was permitted immediately after discharge from hospital, whereas return to hard work applying severe burden to a waist was often permitted 4-6 weeks after surgery for preventing recurrence of disc herniation.

We reviewed type of occupation, disease, operated intervertebral level, type of surgery, surgical time, surgery-related complications, and time to RTW. As the parameter of surgical invasiveness to back muscle in the early postoperative period, the values of C-reactive protein (CRP) 1 or 2 day after surgery were collected from this series.

Lewandrowski et al. ${ }^{7}$ classified the type of physical work according to energy consumption per min (Kcal/min) as Light, Medium, Heavy, and Very Heavy using guidelines adopted from the US Department of Labor published in the online Dictionary of Occupational Titles. In the present study, the time to RTW was also reviewed based on this classification. Furthermore, comparison of the patients with quick RTW within 7 days (quick RTW group) and late RTW of more than 30 days (late RTW group) was performed to investigate the factors that shorten or delay the time to RTW. Analyzed data for comparison of two groups included age, sex, operated intervertebral level, presence of deformity, type of surgery, surgical time, and type of work classified as described above. In addition, for the assessment of clinical outcome, visual analog scale (VAS) before and after surgery of two groups was analyzed.

Statistical analysis was done using SPSS version 21.0 software (IBM Corp., Armonk, NY, USA). For continuous variables, the data are shown as mean and standard deviation values for normally distributed data and as median and interquartile range (IQR) values for non-normally distributed data. For two group comparisons, independent continuous variables were compared using a Student's t-test for normally distributed data and Mann-Whitney test for non-normally distributed data. Categorical variables were compared using the chi-square test. $P<0.05$ was considered significant.

\section{Results}

Occupation types varied and included physician, nurse, helper, clerk, construction worker, cook, school teacher, etc. Discectomy was performed for 48 HNP cases and for the remaining 2 LSS cases, ventral facetectomy was performed. The total number of the operated intervertebral levels was 52 and the distribution was as follows; $33(63 \%)$ at L4/5, 12 $(23 \%)$ at L5/S, and $7(13 \%)$ at L3/4. Two patients of those underwent double-level TF-FES surgery. Mean surgical time was $77.0 \pm 19.4$ minutes. There were no surgery-related complications including exiting nerve root injury, dural tear, hematoma, and infection. The laboratory data of CRP 1 or 2 day after surgery could be collected from 39 of 50 patients in this series. Median value of CRP was $0.13 \mathrm{mg} / \mathrm{L}$ (IQR: $0.08-0.25 \mathrm{mg} / \mathrm{L}$ ). Those data were nearly normal value and indicated very less invasiveness of TF-FES surgery to back muscle.

The time to RTW varied from 2 to 150 days. Figure 1 indicates the distribution of the cases with reference to time to RTW. Median time to RTW was 21 days (IQR: 9-39 days). Thus, about half of the patients $(26 / 50)$ achieved RTW within 14 days, and surprisingly, 12 cases (24\%) could have RTW within 7 days. Also, 92\% (46/50) achieved RTW within 3 months, and $100 \%(50 / 50)$ cases achieved RTW within 5 months.

Table 1 presents the patients characteristics of quick RTW group $(\mathrm{n}=12)$ and late RTW group $(n=13)$. There was a significant difference in the sex ratio $(P=0.035<0.05)$, whereas age, operated intervertebral level, presence of deformity, type of surgery, and surgical time were not significantly different between groups.

Occupations in the quick RTW group included physician, office worker, and bar owner. Most had substantial responsibility at work and could not take prolonged sick leave due to their position. 11 (92\%) were in the Light work and 1 (8\%) was in Medium work. None were in the Heavy or Very heavy work group. Occupations in the late RTW group included nurse, helper, and office worker. Seven (54\%) were in the Light work and six (46\%) were Medium work. None were in the Heavy or Very heavy work. In the quick RTW group, there were significantly more patients in the Light work than in the late RTW group $(P=0.035<0.05)$. These findings suggest that 


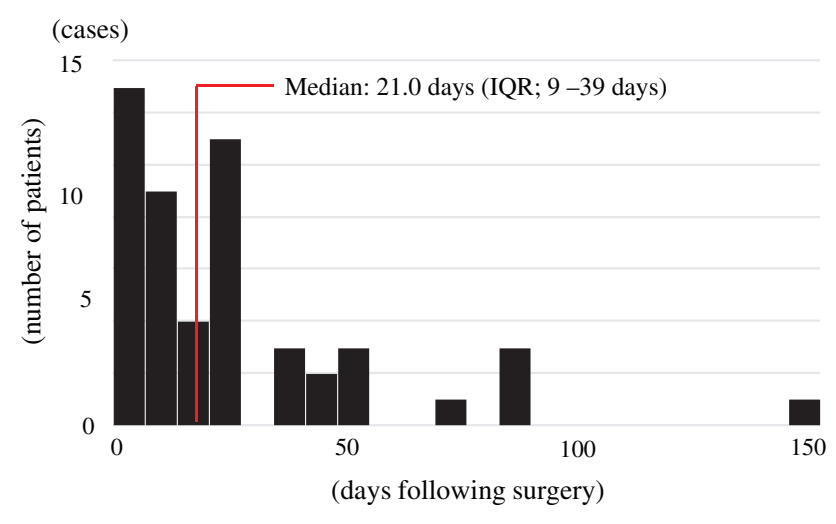

Fig. 1 Bar graph shows the distribution of cases according to the time to RTW. RTW: return to work.

patients could RTW quickly because they were engaged in light work activity. Also, they could not take a prolonged sick leave due to their positions, so this may also account for the quick RTW.

From 15 of 50 patients in this study, VAS for low back pain, leg pain, and leg numbness before and after surgery could be analyzed (Table 2). Mean follow-up period of the quick RTW group $(\mathrm{n}=5)$ and the late RTW group $(\mathrm{n}=10)$ were $3.1 \pm 1.7$ and $4.5 \pm 3.4$ months, respectively. No significant difference was observed between the two groups statistically in the preoperative and postoperative VAS, and the improvement rate of VAS.

\section{Case Presentation}

A 57-year-old man who was a board member of a prefectural committee had persistent leg and back pain despite conservative treatment for more than 6 months. His work involved frequent important meetings, and he could not afford to be absent. Subsequently, he developed weakness of the first toe and started limping. He was referred to us for further management with minimally invasive surgery because he could not take prolonged sick leave.

Neurological examination revealed a right L5 radiculopathy with the following findings. Numbness was noted along the right L5 dermatome. Manual Motor Testing (MMT) score for the extensor hallucis longus (EHL) was $2 / 5$ at the right side. Testing for signs of sciatic nerve tension using the straight leg raising test was positive at 30 degrees on the right side. Figure 2A shows the magnetic resonance imaging (MRI) scans before surgery. Sagittal imaging revealed $\mathrm{HNP}$ at L3/4 and L4/5. Symptoms suggested L5 radiculopathy; thus, L3/4 HNP would not be related to the symptom. Axial MRI at L4/5 showed right L5 nerve root impingement by HNP. Hypertrophy of the facet joint caused lateral recess stenosis, and the pathology was even more obvious on computed tomography (CT) scans, particularly on the axial scan (Fig. 2B).

Based on a diagnosis of L5 radiculopathy due to HNP with lateral recess stenosis at right L4/5, he underwent full-endoscopic decompression surgery including discectomy and ventral facetectomy under local anesthesia on a Wednesday. HNP was successfully removed after ventral facetectomy, and complete decompression of the L5 nerve root was confirmed. No surgery-related complications were noted, such as exiting nerve root injury, hematoma, or surgical site infection. Leg pain disappeared immediately, and muscle weakness improved to $3 / 5$ after the surgery. He was able to attend an important work meeting the following Monday. His time to RTW was just at 5 days after the surgery. Two months after the surgery, MMT of the EHL improved to $4 / 5$ and he was no longer limping. Two years after the surgery, he had no symptoms, and clinical outcome was excellent.

Figure 3A shows the MRI scans after the surgery. $\mathrm{HNP}$ has been removed and the lateral recess is decompressed. The sagittal CT scan (Fig. 3B, left) shows complete removal of the superior articular process; however, facet contact persists between the interior articular process and the lamina. The axial scan clearly shows ventral facetectomy and bony decompression in the right lateral recess at L4/5 (Fig. 3B, middle). The three-dimensional CT scan (Fig. 3B, right) shows the intervertebral foramen is clearly opened up in comparison with findings in Fig. 2B.

\section{Discussion}

The biggest merit of TF-FES surgery is that it is minimally invasive for the muscles of the back. It can also be performed under local anesthesia. In this study, we analyzed the timing of RTW after TF-FES according to occupational type. Our data showed that median time to RTW was 21 days (IQR: 9-39 days) and $23.5 \%$ of our cases could have RTW within 7 days. That results were comparatively quicker than the times reported in reports on traditional spine surgery. Birkenmaier et al. ${ }^{8)}$ reported that full-endoscopic spine surgery could have faster RTW than microscopic surgery, and Ruetten et al. ${ }^{9)}$ compared time until RTW between endoscopic surgery and microscopic surgery and found that it was shorter after the endoscopic technique (25 days vs. 49 days, respectively). Mahesha ${ }^{10)}$ reported very similar results to ours, with a mean time to RTW of 36 days (range: $2-100$ days). Khan et al. ${ }^{11)}$ analyzed 12,435 cases after traditional lumbar surgery for degenerative disease. In total, 9,768 (78.6\%) patients achieved RTW within 3 months and 10,604 (85.3\%) 
Table 1 Comparison of the patients with quick RTW and late RTW groups

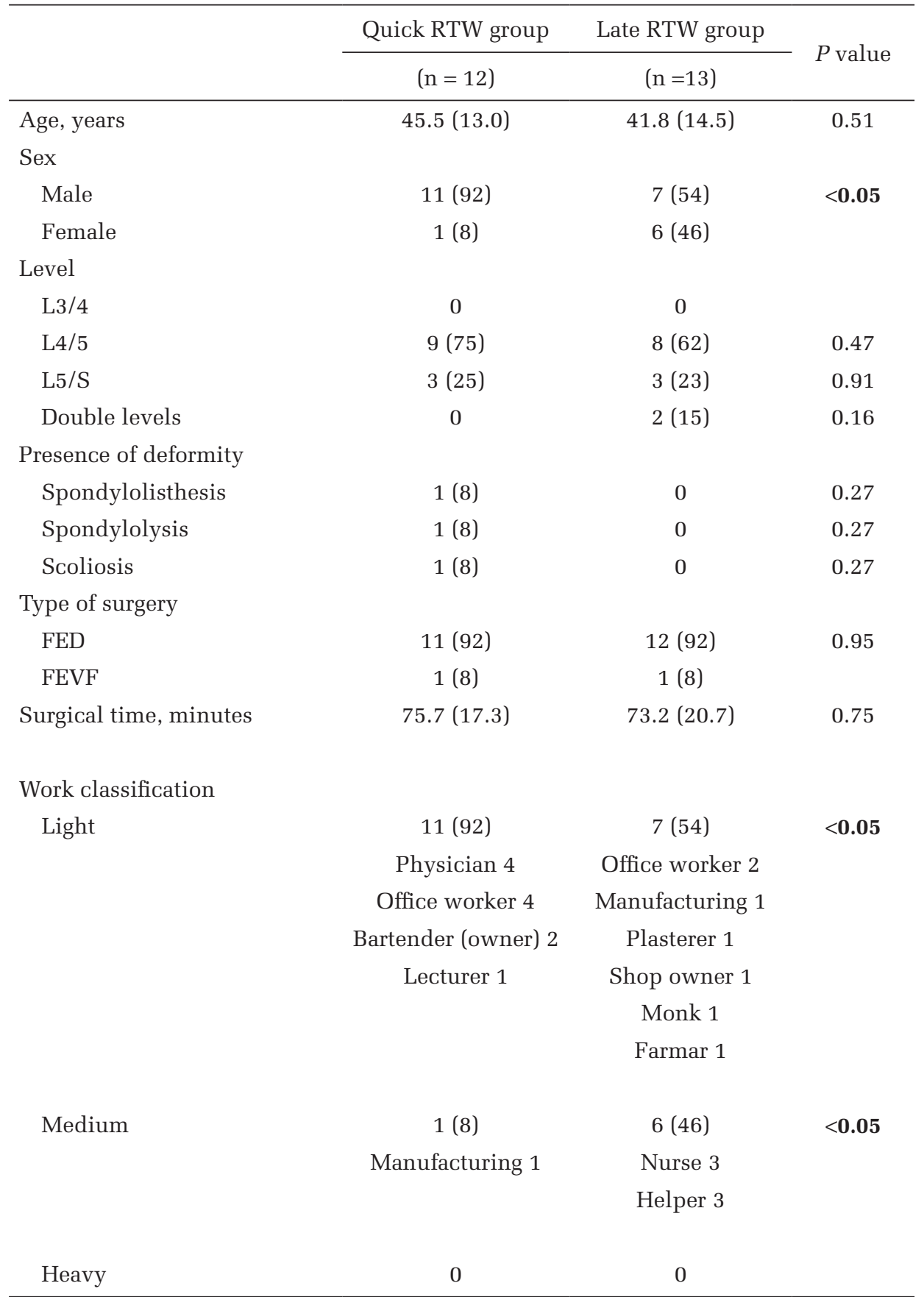

Data are presented as mean (SD), median (IQR) and n (\%). FED: full-endoscopic discectomy, FEVF: full-endoscopic ventral facetectomy, IQR: interquartile range, RTW: return to work.

achieved it within 12 months. In our series, 92.0\% of patients (46/50) achieved RTW within 3 months and $100 \%$ achieved it within 5 months.

The elevation of CRP level has been widely recognized in the postoperative phase, typically peaks between 24 and 72 hours, and is reflecting the postoperative inflammatory reactions and response to tissue damage. Sasaoka et al. ${ }^{12)}$ presented the data that the mean CRP level 1 day postoperatively were $0.91 \mathrm{mg} / \mathrm{dL}$ in the microendoscopic discectomy, $1.76 \mathrm{mg} / \mathrm{dL}$ in the microdiscectomy, and $5.44 \mathrm{mg} /$ $\mathrm{dL}$ in the open laminotomy. In our study, median value of CRP was $0.13 \mathrm{mg} / \mathrm{L} 1$ or 2 days after surgery. There was nearly no elevation of the values of CRP in the early period after TF-FES surgery. That result might verify less invasiveness of TF-FES 
Table 2 Comparison of VAS between patients with quick RTW and late RTW groups

\begin{tabular}{lccc}
\hline & $\begin{array}{c}\text { Quick RTW group } \\
(\mathrm{n}=5)\end{array}$ & $\begin{array}{c}\text { Late RTW group } \\
(\mathrm{n}=10)\end{array}$ & P value \\
\hline Low back pain & $6.0(1.2)$ & $5.3(2.9)$ & 0.59 \\
$\quad$ Pre, cm & $2.6(2.4)$ & $3.8(3.3)$ & 0.49 \\
Post, cm & $59.0(31.7)$ & $39.1(35.4)$ & 0.29 \\
$\quad$ Improvement rate, \% & $6.0(2.3)$ & $5.2(3.0)$ & 0.61 \\
Leg pain & $2.2(1.9)$ & $3.1(3.0)$ & 0.57 \\
$\quad$ Pre, cm & $53.2(41.3)$ & $45.2(42.5)$ & 0.73 \\
$\quad$ Post, cm & & & \\
Improvement rate, \% & $5.6(2.6)$ & $7.9(3.9-8.0)$ & 0.80 \\
Leg numbness & $1.8(1.9)$ & $1.0(0.3-3.7)$ & 0.95 \\
$\quad$ Pre, cm & $75.7(27.3)$ & $75.0(12.5-87.0)$ & 0.36 \\
$\quad$ Post, cm & $3.1(1.7)$ & $4.5(3.4)$ & 0.41 \\
$\quad$ Improvement rate, \% & & & \\
Follow-up period, months & & & \\
\hline
\end{tabular}

Data are presented as mean (SD) or median (IQR). IQR: interquartile range, Pre: preoperatively, Post: postoperatively, RTW: return to work, VAS: visual analog scale.

surgery, and the patients who underwent TF-FES surgery were expected to be quickly able to return to society without pain due to tissue damage. Therefore, full-endoscopic lumbar surgery would be preferable to traditional spine surgery in terms of quicker RTW.

According to a report by Lewandrowski et al., ${ }^{7)}$ the surgery-related complication of dorsal root ganglion irritation (exiting nerve root injury) would be a risk factor for delayed RTW. In our series, none of our patients developed complications. In the previous report on surgery-related complications of full-endoscopic surgery, exiting nerve root injury was found in $2 \%$ of cases. ${ }^{13)}$ At that time, all surgeries were typically performed as an "inside-out procedure"6) that was done by $8 \mathrm{~mm}$ first inserting cannula into the disc just underneath the HNP and removing the HNP fragment. Currently, an "outside-in procedure" as mentioned in the materials and methods section is proposed as safer and was useful for preventing exiting nerve root injury ${ }^{6}{ }^{6}$ Based on this, we opted to perform the outside-in procedure, and in this case series between January 2016 and April 2018 we found no complications. The absence of complications will facilitate a quicker RTW.

In our review of 12 patients with a quick RTW of within 7 days, our findings highlight two factors: doing Light physical work and working in a position of substantial responsibility with inability to take prolonged sick leave. Lewandrowski et al. ${ }^{7)}$ retrospectively analyzed 442 cases after full-endoscopic spine surgery and found the Light work group had the quickest time to RTW (mean and median days) compared with the Medium and Heavy work groups. Our results are in good agreement, as we had 12 cases with quick RTW (within 7 days), 11 involving Light work. Taken together, these findings suggest that patients who perform Light work should be able to achieve RTW quickly following TF-FES.

For certain occupational types, prolonged sick leave is not possible because of work responsibilities. We found that the majority of our patients in the quick RTW group (RTW within 7 days) had such occupation types and we described a representative case of a 57-year-old man with a work responsibilities who was able to attend an important work meeting just 5 days after the surgery.

Sugiura et al. ${ }^{14)}$ reported RTW of 5 days for a 60-year-old dentist who underwent simultaneous bilateral full-endoscopic decompression of lumbar lateral recess stenosis via a transforaminal approach under local anesthesia, concluding that FES under local anesthesia is effective for prompt return to the original work activities. Similarly, Fujii et al. ${ }^{15}$ stated the importance of FES for quick RTW in a study where five of six physicians had a quick RTW within a week, with the remaining physician (a resident) permitted to take 2 weeks of sick leave.

Taking together these previous findings and ours, we conclude that TF-FES under local anesthesia can be very useful in terms of prompt RTW. In particular, TF-TFS offers a clear benefit for patients 


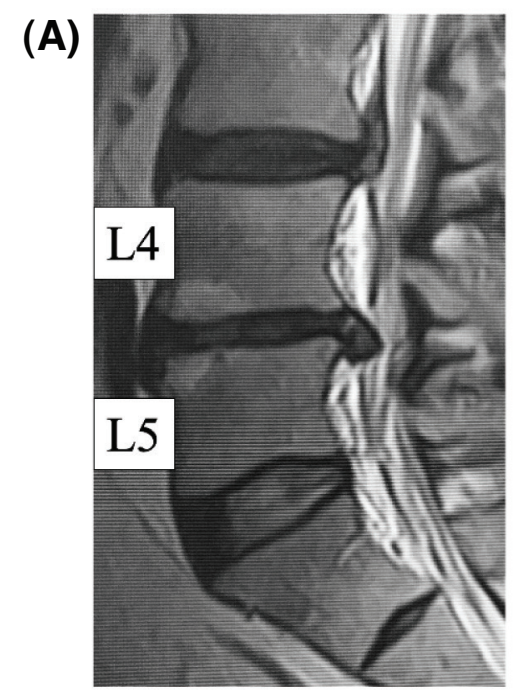

Right para-sagittal

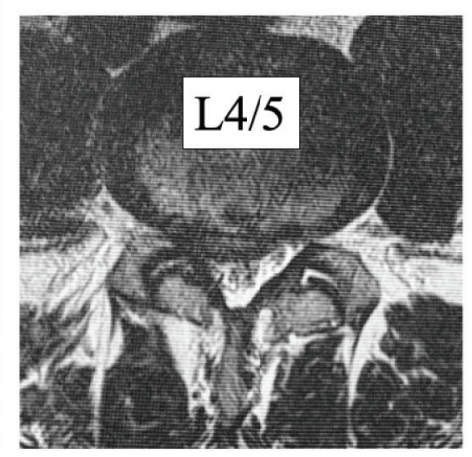

Axial atL4/5

(B)

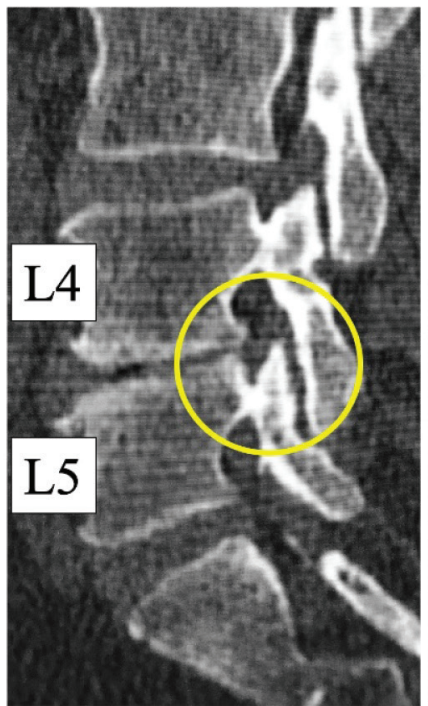

Right para-sagittal

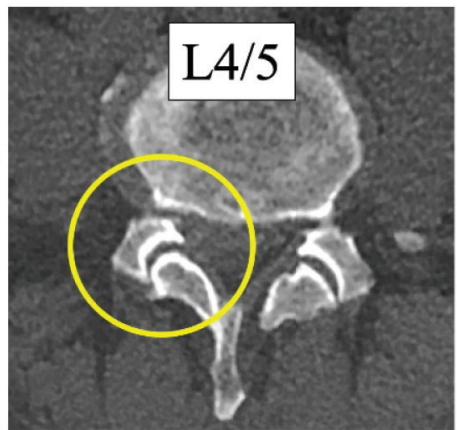

Axial atL4/5

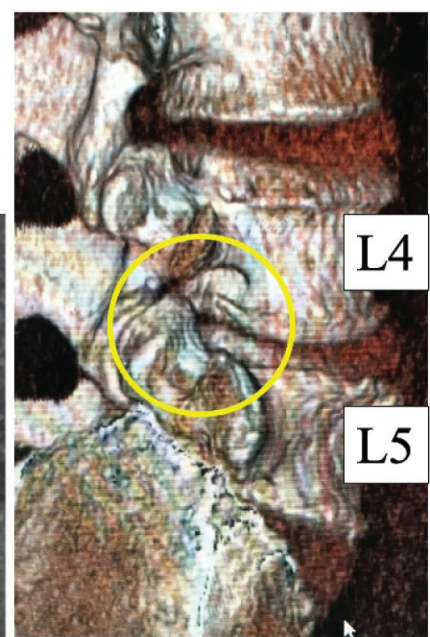

3 dimensional CT

Fig. 2 Preoperative images. (A) MRI scans before surgery. Left panel: Right sagittal image showing HNP at L3/4 and L4/5. Right panel: axial image at L4/5 showing right L5 nerve root impingement by HNP. (B) CT scans before surgery. Hypertrophy of the facet joint causing lateral recess stenosis, especially on the middle panel. CT: computed tomography, HNP: herniated nucleus pulposus, MRI: magnetic resonance imaging.

who cannot take prolonged sick leave due to the demands of their work.

On the other hand, 13 patients $(25.5 \%)$ needed more than a month for RTW in spite of minimally invasive surgical treatment. Based on our findings, the patients who were female and in the Medium work were thought to be late for RTW. The reason for late RTW was probably that the patients in the Medium work were stopped returning to work by attending physician for preventing recurrence of disc herniation, and they were relatively easy to take sick leave because of the work types like nurse or helper, etc.
Some of the limitations of this study are the following. First, more objective data such as less elevation of low back pain in the period between surgery and RTW would be necessarily to present that less invasiveness of TF-FES surgery was related to quick RTW. Therefore, further research is needed in the future. Second, we included a small number of patients in comparison of quick and late RTW group, which may contribute to the bias in statistics. Presently, collecting data samples is still continuing in our institute. Third, since most of the patients who underwent TF-FES surgery might have desires to RTW quickly as soon 


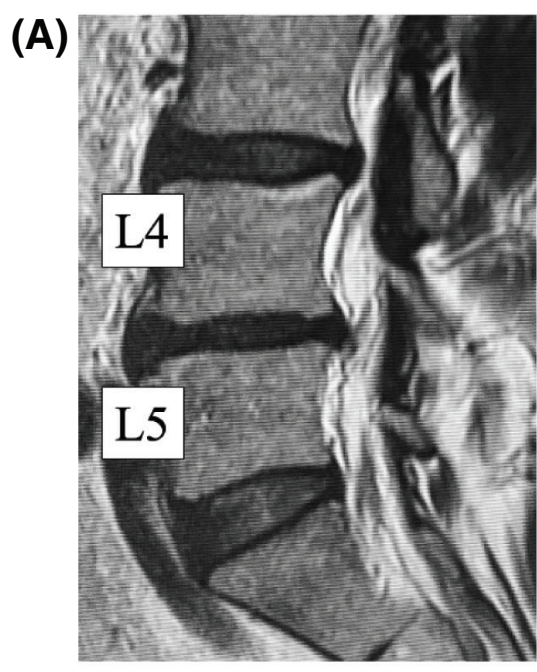

Right para-sagittal

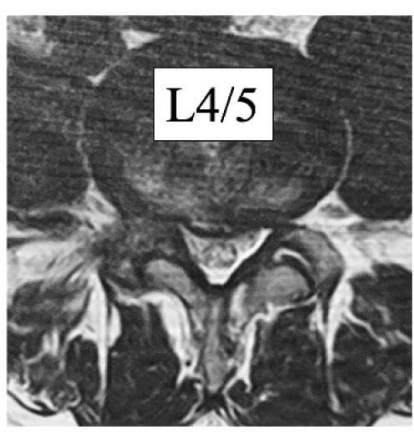

Axial at L4/5

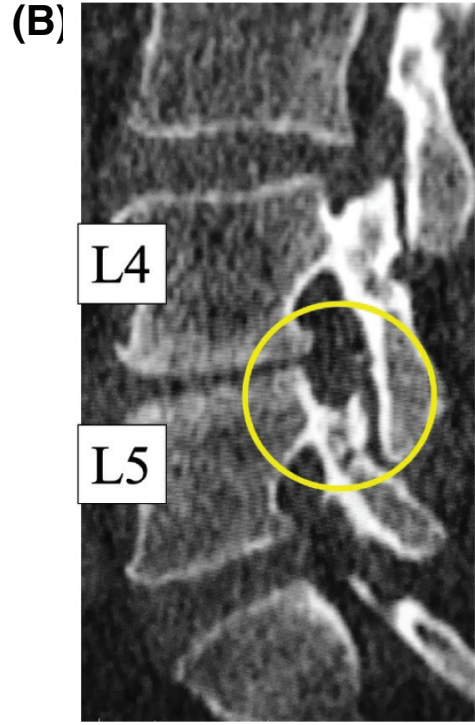

Right para-sagittal

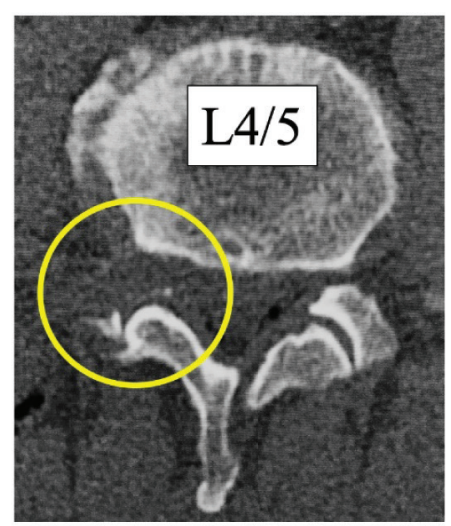

Axial at L4/5

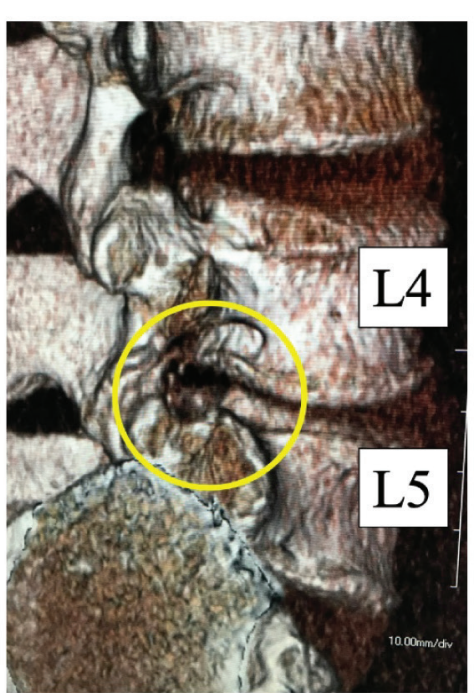

3 dimensional CT

Fig. 3 Postoperative images. (A) MRI scans after surgery. HNP has been removed and the lateral recess is opened-up. (B) CT scans after surgery. Left panel: sagittal CT scan showing complete removal of the superior articular process; however, facet contact persists between the interior articular process and lamina. Middle panel: Axial scan clearly showing ventral facetectomy and bony decompression in the right lateral recess at L4/5. Right panel: Three-dimensional CT scan showing patency of intervertebral foramen, with obvious patency compared with the findings in Fig. 2B. CT: computed tomography, HNP: herniated nucleus pulposus, MRI: magnetic resonance imaging.

as possible, they achieved RTW quicker than the general population. That may cause a selection bias. The solution to those limitations was difficult because of retrospective study design.

\section{Conflicts of Interest Disclosure}

The authors declare no conflicts of interest associated with this manuscript.

\section{References}

1) Recommendation by the Loyal College of Surgeons. https://www.rcseng.ac.uk/patient-care/recovering-from-surgery/discectomy/returning-to-work/

2) Yeung AT: The evolution of percutaneous spinal endoscopy and discectomy: state of the art. Mt Sinai J Med 67: 327-332, 2000

3) Yeung AT, Tsou PM: Posterolateral endoscopic excision for lumbar disc herniation: Surgical 
technique, outcome, and complications in 307 consecutive cases. Spine (Phila Pa 1976) 27: 722-731, 2002

4) Sairyo K, Nagamachi A: State-of-the-art management of low back pain in athletes: instructional lecture. J Orthop Sci 21: 263-272, 2016

5) Sairyo K, Chikawa T, Nagamachi A: State-ofthe-art transforaminal percutaneous endoscopic lumbar surgery under local anesthesia: discectomy, foraminoplasty, and ventral facetectomy. J Orthop Sci 23: 229-236, 2018

6) Yoshinari H, Tezuka F, Yamashita K, et al: Transforaminal full-endoscopic lumbar discectomy under local anesthesia in awake and aware conditions: the inside-out and outside-in techniques. Curr Rev Musculoskelet Med 12: 311-317, 2019

7) Lewandrowski KU, Ransom NA, Yeung A: Return to work and recovery time analysis after outpatient endoscopic lumbar transforaminal decompression surgery. J Spine Surg 6: S100-115, 2020

8) Birkenmaier C, Komp M, Leu HF, Wegener B, Ruetten S: The current state of endoscopic disc surgery: review of controlled studies comparing full-endoscopic procedures for disc herniations to standard procedures. Pain Physician 16: 335-344, 2013

9) Ruetten S, Komp M, Merk H, Godolias G: Fullendoscopic interlaminar and transforaminal lumbar discectomy versus conventional microsurgical technique: a prospective, randomized, controlled study. Spine (Phila Pa 1976) 33: 931-939, 2008
10) Mahesha K: Percutaneous endoscopic lumbar discectomy: Results of first 100 cases. Indian J Orthop 51: 36-42, 2017

11) Khan I, Bydon M, Archer KR, et al.: Impact of occupational characteristics on return to work for employed patients after elective lumbar spine surgery. Spine $J$ 19: 1969-1976, 2019

12) Sasaoka R, Nakamura H, Konishi S, et al.: Objective assessment of reduced invasiveness in MED. Compared with conventional one-level laminotomy. Eur Spine J 15: 577-582, 2006

13) Sairyo K, Matsuura T, Higashino K, et al.: Surgery related complications in percutaneous endoscopic lumbar discectomy under local anesthesia. J Med Invest 61: 264-269, 2014

14) Sugiura K, Yamashita K, Manabe H, et al.: Prompt return to work after bilateral transforaminal fullendoscopic lateral recess decompression under local anesthesia: a case report. J Neurol Surg (A), in press

15) Fujii Y, Yamashita K, Sugiura K, et al.: Quick return to the original activity from the lumbar spinal disorders by the minimally invasive percutaneous endoscopic decompression surgery for the medical doctors. J Spine Surg 6: 294-299, 2020

Corresponding author: Koichi Sairyo, MD, PhD Department of Orthopedics, Tokushima University Graduate School, 3-18-15 Kuramoto, Tokushima, Tokushia 770-8503, Japan. e-mail: sairyokun@gmail.com 